\title{
Comparative study between pSWE and 2D-SWE techniques integrated in the same ultrasound machine, with Transient Elastography as the reference method
}

\author{
Camelia Foncea, Alina Popescu, Raluca Lupușoru, Renata Fofiu, Roxana Șirli, Mirela \\ Danilă, Ioan Sporea
}

Department of Gastroenterology and Hepatology, „Victor Babeș” University of Medicine and Pharmacy, Timișoara, Romania

\begin{abstract}
Aim: To evaluate the feasibility of two elastographic methods, point Shear Wave Elastography (pSWE) and two dimensional Shear Wave Elastography (2D-SWE), integrated in the same ultrasound machine, for liver fibrosis (LF) assessment, using Transient Elastography (TE) as the reference method. Material and methods: We included in the study 115 subjects in which LF was evaluated in the same session by TE (FibroScan, EchoSens), pSWE and 2D-SWE (Samsung-Medison RS85). Reliable liver stiffness (LS) measurements were defined: for TE the median value of 10 measurements with interquartile range $(\mathrm{IQR} / \mathrm{M}) \leq 30 \%$, while for $\mathrm{pSWE}$ and $2 \mathrm{D}$-SWE the median value of 10 measurements, with a reliability measurement index $(\mathrm{RMI}) \geq 0.5$ and $\mathrm{IQR} / \mathrm{M} \leq 30 \%$. For classification of LF severity we used TE as the reference method with the following cut-offs: $F 2 \geq 7 \mathrm{kPa}, \mathrm{F} 3 \geq 9.5 \mathrm{kPa}$ and F4 $\geq 12 \mathrm{kPa}$. Results: Reliable measurements by TE were obtained in $98.2 \%$ of cases $(113 / 115)$, by pSWE in $93.9 \%$ of cases (108/115) and by 2D-SWE in $92.1 \%$ of cases (106/115), so the final analysis included 101 patients. We divided the cohort into 3 groups: fibrosis $<$ F2 (66.3\%), fibrosis F2-3 (15.8\%) and F4 (17.8\%). The cut-off values calculated for $\mathrm{pSWE}$ were: $\mathrm{F} \geq 2 \mathrm{LS}>5.9 \mathrm{kPa}$ [AUROC $=0.95,95 \% \mathrm{CI}(0.89 ; 0.98), \mathrm{p}<0.0001, \mathrm{Se}=94.1 \%, \mathrm{Sp}=89.5 \%$, $\mathrm{PPV}=82.1 \%, \mathrm{NPV}=96.8 \%$ ]; F4 LS $>8 \mathrm{kPa}$ [AUROC=0.98, 95\%CI $(0.94 ; 0.99), \mathrm{p}<0.0001, \mathrm{Se}=94.4 \%, \mathrm{Sp}=95.1 \%, \mathrm{PPV}=81 \%$, $\mathrm{NPV}=98.7 \%$, while for 2D-SWE they were: $\mathrm{F} \geq 2 \mathrm{LS}>6.1 \mathrm{kPa}[\mathrm{AUROC}=0.93,95 \% \mathrm{CI}(0.86 ; 0.97), \mathrm{p}<0.0001, \mathrm{Se}=91.1 \%$, $\mathrm{Sp}=80.6 \%, \mathrm{PPV}=70.5 \%, \mathrm{NPV}=94.7 \%] ; \mathrm{F} 4 \mathrm{LS}>7.6 \mathrm{kPa}[\mathrm{AUROC}=0.98,95 \% \mathrm{CI}(0.93 ; 0.99), \mathrm{p}<0.0001, \mathrm{Se}=100 \%, \mathrm{Sp}=91.5 \%$, $\mathrm{PPV}=72 \%, \mathrm{NPV}=100 \%$ ]. We observed strong correlations between LS values obtained by TE and 2D-SWE ( $\mathrm{r}=0.85$ ), between TE and pSWE $(r=0.88)$ and between pSWE and 2D-SWE $(r=0.90)(p=0.37)$, respectively. There were no significant differences between the mean values obtained by pSWE and 2D-SWE ( $\mathrm{p}=0.96)$. Conclusion: The pSWE and 2D-SWE are feasible methods for assessing liver fibrosis, both techniques strongly correlating with TE results.
\end{abstract}

Keywords: liver fibrosis; elastography; pSWE; 2D-SWE; Transient Elastography

\section{Introduction}

Liver fibrosis is a progressive process and the first step in developing liver cirrhosis. There are many causes that can lead to liver fibrosis, but the most frequent re-

Received 28.09.2019 Accepted 02.12.2019

Med Ultrason

2020, Vol. 22, No 1, 13-19

Corresponding author: Alina Popescu

8/7 Barbu Iscovescu street,

300561, Timișoara, Romania

E-mail: Alinamircea.popescu@gmail.com main chronic viral hepatitis (B or C viruses), excessive alcohol consumption (alcoholic steatohepatitis - ASH) and non-alcoholic fatty liver disease (NAFLD).

It is important to have useful tool for the diagnosis, management, treatment and follow-up of these diseases. Several methods can be used in the assessment of liver fibrosis. The „gold standard" method is liver biopsy (LB), an invasive procedure with some drawbacks: potential complications, sampling errors, high costs, etc. [1]. Due to these facts, it is not always accepted by patients, especially if needed to be repeated for disease follow-up. Thus, the rate of LB seems to decrease, being replaced by non-invasive techniques [2]. 
Non-invasive methods for the assessment of liver fibrosis are either biological [3] or elastographic (ultrasound based or magnetic resonance imaging based). According to the European Federation of Societies for Ultrasound in Medicine and Biology (EFSUMB) and World Federation for Ultrasound in Medicine and Biology (WFUMB) Guidelines, the ultrasound-based elastographic methods are divided into strain and shear waves elastography (SWE) techniques [4,5]. The last category includes Transient Elastography (TE), point Shear Wave Elastography (pSWE) and bidimensional Shear Wave Elastography (2D-SWE).

The first elastographic method used for liver fibrosis assessment was Transient Elastography (TE) and afterwards other techniques became available, such as pSWE and 2D-SWE. TE technique is accepted by international guidelines for the evaluation of liver fibrosis [6], but it has some limitations related to the presence of: ascites, over weight and the lack of gray scale ultrasound guidance [4,5]. pSWE and 2D-SWE are integrated into ultrasound machines, allowing real-time visualization of liver structure and having the ability to choose the region of interest, avoiding vessels and the liver capsule. They are also feasible in patients with ascites [4,5]. Nowadays, pSWE and 2D-SWE are implemented in platforms developed by several vendors (Supersonic Image, Philips, General Electric Healthcare, Siemens, Canon/Toshiba, Samsung, etc.) and, in some cases, both techniques are integrated in the same ultrasound machine (for example Philips, Samsung).

The aim of our study was to evaluate the feasibility and usefulness of two elastographic techniques (pSWE and 2D-SWE) integrated in the same ultrasound machine (Samsung-Medison RS85), for liver fibrosis assessment, using TE as the reference method.

\section{Material and methods}

\section{Subjects}

A prospective study was performed including 115 subjects, with or without chronic liver disease: 31 healthy volunteers and 84 patients with previously diagnosed chronic liver disease. Inclusion criteria for healthy subjects were: no history of liver disease, normal liver function tests, negative $\mathrm{HBV} / \mathrm{HCV}$ infection, normal ultrasound aspect of the liver and liver stiffness (LS) values evaluated by $\mathrm{TE}<5 \mathrm{kPa}$ [5]. The inclusion criteria for patients with chronic liver disease were: history of chronic viral hepatitis (HBV or HCV), alcoholic or nonalcoholic steatohepatitis, or liver cirrhosis; aminotransferases less than 3 times the upper level of normal (ULN). The exclusion criteria were: presence of ascites, aminotrans- ferases higher than $3 x U L N$, signs of biliary obstruction or liver congestion and focal liver lesions found during ultrasound examination. In all patients, the following parameters were documented: age, gender, body mass index (BMI), presence of chronic hepatopathies, presence of liver cirrhosis.

All subjects included agreed to undergo elastographic measurements and the study was approved by the Ethics Committee and was performed in accordance with the last revised version of the Helsinki Declaration.

LS measurements were obtained in all subjects in the same session by means of all three elastographic techniques: TE, pSWE and 2D-SWE, the last two integrated in the same ultrasound machine (Samsung-Medison RS85). Fourteen patients were excluded from the study cohort due to unreliable LS measurements by TE or SWE, so the final analysis included 101 subjects.

\section{Elastographic techniques:}

All elastographic measurements were performed in the same session, in fasting conditions, with the patients in supine position and the right arm in maximum abduction, by two operators with at least one-year experience in ultrasound and liver elastography. The right liver lobe was examined through an intercostal space approach.

TE was performed with FibroScan ${ }^{\circledR}$ device (EchoSens, Paris, France), which incorporates an ultrasound transducer probe mounted on the axis of a vibrator. Ten valid measurements were performed in each patient by the $\mathrm{M}$ or XL probe, and the median value was calculated. Reliable LS measurements were defined as follows: the median value of 10 measurements with an interquartile range $(\mathrm{IQR} / \mathrm{M}=$ the difference between the 75th and 25th percentile, actually the range of the middle $50 \%$ of the data) $\leq 30 \%$, and the results were expressed in kilopascals $(\mathrm{kPa})$. Unreliable LS measurements were defined by $\mathrm{IQR} / \mathrm{M} \geq 30 \%[7,8]$. For the classification of LF severity we used TE as the reference method, with the following cut-offs used in the Tsochatzis meta-analysis: F2 $\geq 7 \mathrm{kPa}$, $\mathrm{F} 3 \geq 9.5 \mathrm{kPa}$ and $\mathrm{F} 4 \geq 12 \mathrm{kPa}[9]$.

pSWE of the liver (S-Shearwave) on Samsung's Medison RS85 was conducted by using first B-Mode imaging, to locate the area of interest, using the CA3-10A convex probe. The Region of Interest (ROI) box was then placed in an area of the right lobe of liver, at least 1-2 $\mathrm{cm}$ below the capsule, avoiding large vessels, and several $\mathrm{S}$-Shearwave measurements were performed with the patient in suspended intermediate respiration.

Additionally, the Reliability Measurement Index (RMI) and Variation Range (VR) were provided in the S-Shearwave Profile. The RMI is a quality control parameter calculated by the weighted sum of two factors: the residual of the wave equation and the magnitude of 
the shear wave. Therefore, high RMI values are strongly correlated with reproducible measurements. A RMI of 0.0 indicates significant error, whereas an RMI of 1.0 indicates no error. Measurements with $\mathrm{RMI}<0.4$ were removed in order to increase reliability. The system automatically calculates the median value and the IQR of the valid measurements [10]. Reliable LS measurements were defined as the median value of 10 measurements, with a $\mathrm{RMI} \geq 0.5$ and $\mathrm{IQR} / \mathrm{M} \leq 30 \%$. 2D-SWE was performed after pSWE, using the same convex CA3-10A probe. A $1 \mathrm{~cm}$ diameter 2D-SWE ROI was placed 1-2 $\mathrm{cm}$ below the liver capsule, avoiding large vessels, in a homogenous region of the color-coded map generated and superimposed by the machine on the B-mode ultrasound image. When a homogenous image was found, the patient was asked to hold the breath in an intermediate position and image acquisition was performed. In 10 seconds of suspended breathing more than 50 frames can be acquired, so there was no need to repeat the procedure. Within each frame, one ROI was placed in order to obtain the measurement. Ten consecutive measurements were performed in different shear wave frames.

Samsung's S-Shearwave Imaging ${ }^{\mathrm{TM}}$ provides an additional image that shows the reliability of elasticity measurements. The index named RMI map helps the user to determine whether the elasticity value has been correctly measured [11]. RMI is calculated by analyzing how much the measured shear wave deviates from the theoretical behavior. Since an image containing both the stiffness map and RMI map is provided by the system, the user can intuitively perform a highly reliable elasticity measurement. Also, RMI-weighted average value within the measured ROI circle is provided. A 2D color-coded RMI image highlights more reliable values with white to yellow colors and less reliable values with red to black colors. The system automatically calculates the median value and IQR of the valid measurements. The same quality criteria were used in order to define reliable measurements: the median value of 10 measurements, with $\mathrm{RMI} \geq 0.5$ and $\mathrm{IQR} / \mathrm{M} \leq 30 \%$ [11].

\section{Statistical analysis}

The statistical analysis was performed using SPSS software v.17 (SPSS Inc., Chicago, IL, USA) and Microsoft Office Excel 2019. The Kolmogorov- Smirnov test was used for testing the distribution of numerical variables. Descriptive statistics was used for clinical, anthropometric and demographic data of patients. $\mathrm{Nu}$ merical variables with normal distribution are presented as means \pm standard deviation, while variables with nonnormal distribution are presented as median values and range. The Kolmogrov-Smirnov test was used for testing the distribution of numerical variables. Qualitative variables were presented as numbers and percentages.

The differences between groups were assessed using the student's t-test for continuous variables with normal distribution, while the Mann-Whitney U's test was used for continuous variables without normal distribution. The Fisher's test and Pearson's chi-squared test were used to compare proportions.

Areas under receiver operating characteristic (AUROC) curves were calculated for pSWE and 2D-SWE measurements from Samsung Medison to identify the cut-off values for various stages of liver fibrosis.

Positive predictive value (PPV - true positive cases/ all positive cases), negative predictive value (NPV - true negative cases/all negative cases) and diagnostic accuracy (sum of true positive and true negative cases/total number of cases) were calculated [12]. 95\% confidence intervals (CI) were calculated for each predictive test and a $p$-value $<0.05$ was considered as significant for each statistical test. For correlations, we used the Sperman test. For comparing the two new techniques (p-SWE and 2D-SWE) with TE we used the Pearson coefficient of precision and accuracy included in the Lin's Concordance Correlation Coefficient (CCC) [13] and the BlandAltman plot analysis.

\section{Results}

Reliable measurements by TE were obtained in $98.2 \%$ (113/115) cases, by pSWE in 93.9\% (108/115) cases and by $2 \mathrm{D}-\mathrm{SWE}$ in $92.1 \%(106 / 115)$ cases, so the final analysis included 101 patients in whom all techniques had reliable measurements. The unreliable results for all the techniques were due to obesity, $\mathrm{p}<0.0001$. The $\mathrm{M}$ probe at TE was used in 72 patients $(71.3 \%)$ and XL probe in 29 patients $(28.7 \%)$.

Considering TE as the reference method to differentiate between stages of fibrosis, using the TE cut-offs proposed by the Tsochatzis meta-analysis [9], the distribution of LF was: F0-1- 67 patients $(66.3 \%), \mathrm{F} 2-3-16$ patients (15.8\%) and $\mathrm{F} 4-18$ patients $(17.8 \%)$.

The patients' characteristics are presented in Table I.

Using TE as the reference method, we calculated the cut-off values for diagnosing significant fibrosis $(\mathrm{F} \geq 2)$ and cirrhosis $(\mathrm{F}=4)$, using Samsung Shearwave methods (Table II). The mean value obtained by pSWE and 2D-SWE were similar: $7.24 \pm 5.88 \mathrm{kPa}$ vs. $7.26 \pm 5.04 \mathrm{kPa}$, $\mathrm{p}=0.96$.

Strong positive correlations between TE measurements and 2D-SWE $(r=0.85)$ and pSWE $(r=0.88)$ and between pSWE and 2D-SWE $(r=0.90)$ were found (Fig 1). 
Table I. Main characteristics of the study group

\begin{tabular}{ll}
\hline Parameter & \\
\hline Age (years)* & $54 \pm 16$ \\
Gender: & \\
$\quad$ male & $51 / 101(50.5 \%)$ \\
female & $50 / 101(49.5 \%)$ \\
BMI (kg/m²)* & $26.3 \pm 5.5$ \\
Diagnosis & \\
$\quad$ Chronic hepatitis B & $15 / 101(14.9 \%)$ \\
Chronic hepatitis C & $21 / 101(20.8 \%)$ \\
NASH/ALD & $33 / 100(32.6 \%)$ \\
$\quad$ Healthy subjects & $32 / 101(31.7 \%)$ \\
Fibrosis stage (FibroScan) & \\
F 0-1 & $67 / 101(66.3 \%)$ \\
F 2-3 & $16 / 101(15.9 \%)$ \\
F=4 & $18 / 101(17.8 \%)$ \\
\hline
\end{tabular}

*Mean \pm standard deviation; BMI - body mass index; NASH - nonalcoholic steatohepatitis; ALD - alcoholic liver disease

According to the Bland-Altman test, the mean difference between TE and p-SWE was $0.9 \pm 0.38$. The $95 \%$ upper and lower limits of agreement (LOA) were 8.5 and $-6.7 \mathrm{kPa}$, respectively (Fig 2a). The mean difference between TE and 2D-SWE was $0.9 \pm 0.4$. The $95 \%$ upper and lower LOA were 9.7 and $-7.9 \mathrm{kPa}$ (Fig 2b), and the mean difference between p-SWE and 2D-SWE was 0.008 2.3 . The $95 \%$ upper and lower LOA were 4.9 and $-4.9 \mathrm{kPa}$, respectively (Fig 2c).

\section{Discussion}

Elastography-based techniques have shown promising results for non-invasive assessment of liver fibrosis $[4,5]$ and nowadays, almost all ultrasound companies have implemented elastography modules in their ultrasound machines. The EFSUMB and WFUMB Elastography Guidelines $[4,5]$ recommend that only Shear Wave Elastography (SWE) should be used in practice for liver fibrosis assessment, considering that strain liver elastography still has limited recommendations in clinical use, at least in Europe. However, all guidelines stipulate that each ultrasound system has its own cut-off values for the various stages of fibrosis and, maybe, for different etiologies of chronic liver disease $[4,5]$. This is why studies are required to find the specific cut-offs values for new devices that are launched on the market.

For a long time, liver biopsy was considered the reference method for the assessment of liver fibrosis, and was used as the gold standard when comparing different elastographic methods. In the last years, we are faced with a dramatic decrease in the number of liver biopsies, mainly because HBV and HCV treatment can be prescribed without a strict evaluation of the severity of fibrosis. In these conditions, assessment of new elastographic methods and systems had to be performed by comparing them with the validated elastographic method, TE. TE was validated by many published papers and meta-analysis and is recommended for liver fibrosis assessment by the EASL Guidelines 2015 [6].

Elastographic methods that use Acoustic Radiation Force Impulse (ARFI) technology (either pSWE or 2DSWE), have several advantages on TE: implementation in an ultrasound machine, allow the direct visualization of interrogated tissue, thus avoiding vessels and can be performed in patients with ascites [4,5]. Published papers on ARFI based elastography show good feasibility and accuracy of the method in LF assessment [14-16]. But the following question arises for practitioners: which method to use in their daily practice?

Published studies compared TE with pSWE (Virtual Touch Quantification -VTQ from Siemens) and 2D-SWE (Aixplorer system from Supersonic Imagine-SSI), considering liver biopsy as a gold standard in patients with chronic liver disease [14] or in patients with NAFLD [15]. A number of 349 consecutive patients with chronic liver diseases were evaluated by means of liver biopsy and non-invasive methods: TE (M and XL probes), VTQ and 2D-SWE.SSI. The three elastographic methods correlated significantly with liver biopsy $(r=0.79$, $\mathrm{p}<0.00001$ for SSI; $\mathrm{r}=0.64, \mathrm{p}<0.00001$ for VTQ and $\mathrm{r}=0.70, \mathrm{p}<0.00001$ for TE respectively). The AUROCs for 2D-SWE.SSI, VTQ and TE for the diagnosis of significant fibrosis $(\geq \mathrm{F} 2)$ were $0.88,0.81$ and 0.84 , respec-

Table II. Predictive values of liver stiffness evaluated by means of Samsung pSWE and 2D-SWE for various stages of fibrosis, using TE as the reference method

\begin{tabular}{llllllll}
\hline Method & Fibrosis stage & Cut-off & Se (\%) & Sp (\%) & PPV (\%) & NPV (\%) & AUROC \\
\hline pSWE & F2 & $>5.9 \mathrm{kPa}$ & 94.1 & 89.5 & 82.1 & 96.8 & $0.95,95 \% \mathrm{CI}(0.89-0.98), \mathrm{p}<0.0001$ \\
& F4 & $>8 \mathrm{kPa}$ & 94.4 & 95.1 & 81 & 98.7 & $0.98,95 \% \mathrm{CI}(0.94 ; 0.99), \mathrm{p}<0.0001$ \\
\multirow{2}{*}{ D-SWE } & F2 & $>6.1 \mathrm{kPa}$ & 91 & 80.6 & 70.5 & 94.7 & $0.93,95 \% \mathrm{CI}(0.86 ; 0.97), \mathrm{p}<0.0001$ \\
& F4 & $>7.6 \mathrm{kPa}$ & 100 & 91.5 & 72 & 100 & $0.98,95 \% \mathrm{CI}(0.93 ; 0.99), \mathrm{p}<0.0001$ \\
\hline
\end{tabular}

AUROC - area under a receiver operating curve; $\mathrm{Se}$ - sensitivity; $\mathrm{Sp}$ - specificity; PPV - positive predictive value; NPV - negative predictive value 

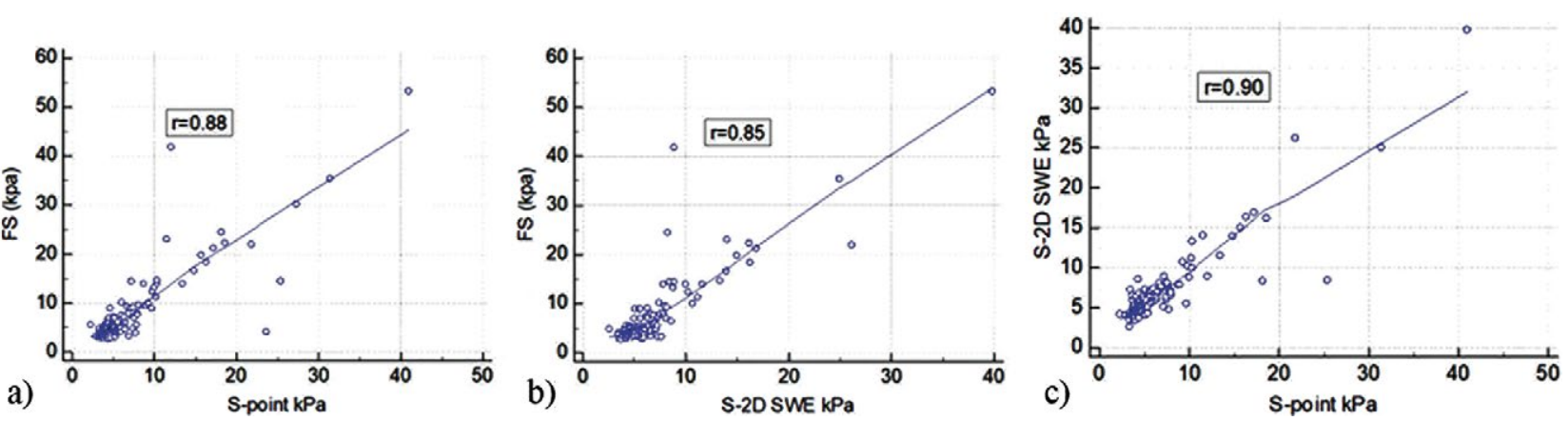

Fig 1. a) Relationship between the mean values assessed by TE and $p$-SWE Samsung, Pearson's correlation coefficient, $r=0.88$; b) Relationship between the mean values assessed by TE and 2D-SWE Samsung, Pearson's correlation coefficient, $r=0.85$; c) Relationship between the mean values assessed by $\mathrm{p}-\mathrm{SWE}$ and 2D- SWE Samsung, Pearson`s correlation coefficient, $\mathrm{r}=0.90$
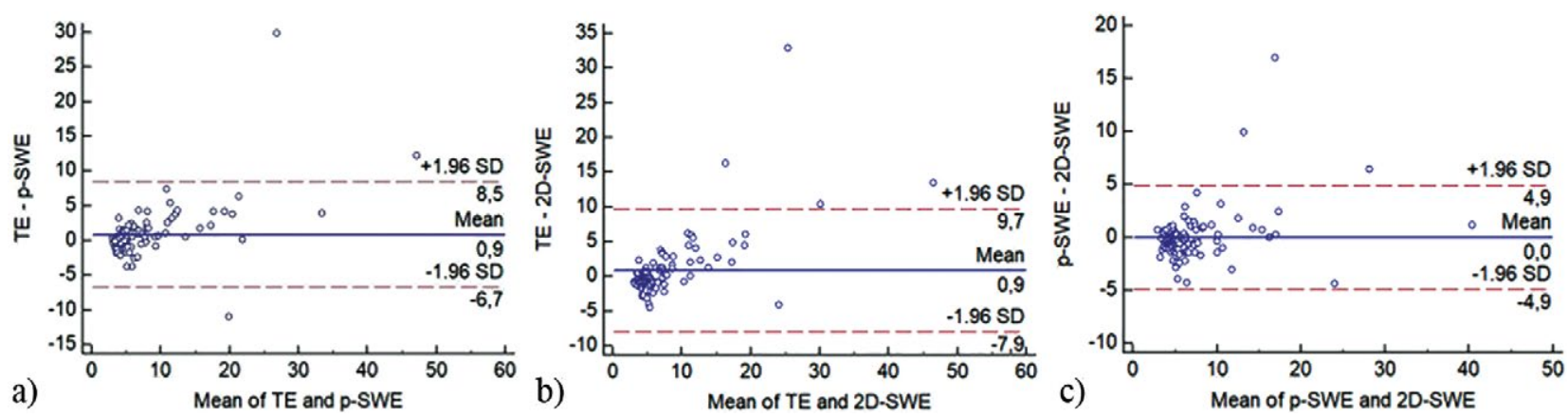

Fig 2. Bland-Altman plots of differences between the elastographic measurements. The solid line represents the mean of the difference between the elastographic methods' measurements taken with the two techniques; the dashed lines define the limit of agreement: a) Comparison between TE and p-SWE. The mean difference is $0.9 \pm 0.38$. The $95 \%$ upper and lower LOA were 8.5 and -6.7 , respectively; b) Comparison between TE and 2D-SWE. The mean difference is $0.9 \pm 0.4$. The $95 \%$ upper and lower LOA were 9.7 and -7.9 , respectively; c) Comparison between 2D-SWE and p-SWE. The mean difference is $0.008 \pm 2.3$. The $95 \%$ upper and lower LOA were 4.9 and -4.9 , respectively.

tively, while for the diagnosis of liver cirrhosis they were $0.93,0.90$ and 0.90 , respectively. Cassinotto et al [15] also compared the same ARFI methods in a group of 291 patients with NAFLD, considering liver biopsy as a reference method, with good AUROC values for the diagnosis of significant liver fibrosis and liver cirrhosis. In both studies, good predictive values for all three methods in assessing the severity of liver fibrosis were obtained. Another published paper [16] showed good correlation between 4 elastographic methods (VTQ, ElastPQ, 2DSWE.SSI and TE) and similar accuracies for the diagnosis of significant fibrosis $(\mathrm{F} \geq 2)$ and liver cirrhosis $(\mathrm{p}>0.05)$.

One of the first published papers regarding S-Shearwave Elastography, using Samsung RS 80A (Samsung Medison) [17] (same p-SWE technique, but different software) compared only the accuracy of p-SWE by Samsung in assessing LS with an established pSWE technique (Virtual Touch Quantification, VTQ) using TE as a reference method. The study included 28 patients (70\% with chronic hepatitis B) who had reliable VTQ, S-
Shearwave and TE measures. The LS value for both exhibited a good, positive correlation $(\mathrm{r}=0.78, \mathrm{p}<0.0001)$. Diagnostic accuracy of different liver fibrosis stages was similar for both techniques. For predicting the presence of severe fibrosis $(\mathrm{F} \geq 3)$ the AUROCs of VTQ $(\mathrm{r}=0.87)$ and $\mathrm{S}$-Shearwave $(\mathrm{r}=0.84)$ were not significantly different $(\mathrm{p}=0.51)$, while for cirrhosis detection $(\mathrm{F}=4)$, were 0.89 , respectively $0.94(\mathrm{p}=0.48)$. The limitations of this study were the small number of patients and cut off values used for staging of liver fibrosis that were proposed for patients with chronic hepatitis B even though the cohort was heterogenous.

A recent multicenter study [18] validated S-shearwave elastography (Samsung RS80A® ultrasound system Samsung Medison) in the assessment of liver fibrosis taking TE and liver biopsy as reference method and included 570 patients, out of which 107 had liver biopsy performed. This study is the first to validate cut off values for S-SWE based on histological assessment. LS measurements on S-SWE (pSWE technique) had a significant correlation with TE values $(\mathrm{r}=0.880, \mathrm{p}<0.001)$. 
The AUROCs of S-SWE for the diagnoses of $\geq F 2$, $\geq F 3$ and F4 were $0.842,0.844$ and 0.850 , respectively and the optimal cut-off values for LS measurements on S-SWE were $>7.0 \mathrm{kPa}$ and $>9.7 \mathrm{kPa}$ for $\geq \mathrm{F} 3$ and F4, respectively. Based on histological findings, S-SWE had the following mean values ( \pm standard deviation) for F0/1, F2, F3, and F4: $4.5( \pm 2.1), 5.9( \pm 2.6), 8.4( \pm 7.3)$, and $14.5( \pm 10.0)$ $\mathrm{kPa}$, respectively $(\mathrm{p}<0.001)$, with a significant positive correlation $(p<0.001)$. These cut-off values are different from our results, the study being performed with the same techniques, but different software.

Another paper, the first published about p-SWE and 2D-SWE performed with Samsung RS85A [19], aimed to evaluate interobserver reproducibility, and accuracy of (SWE) in staging hepatitis $\mathrm{C}$ virus associated liver fibrosis, taking TE as a reference method and included 49 patients with chronic disease and 10 healthy control. Good reproducibility was found between the methods (ICC $=0.91,95 \%$ CI:0.84-0.95, $\mathrm{p}<0.001$ and $\mathrm{ICC}=0.79$, $95 \%$ CI:0.65-0.87, $\mathrm{p}<0.001)$. The final analysis included only 33 patients with hepatitis $\mathrm{C}$ virus and showed good performance in the diagnostic accuracy of clinically significant $(\geq F 2)$ liver fibrosis and liver cirrhosis, AUROC 0.94 and 0.89 , respectively, but it only included the pSWE technique and the number of subjects was relatively small.

All these studies showed comparable results regarding feasibility, sensitivity, specificity, correlation and accuracy for these techniques, similar to our results.

Currently, only a few ultrasound producers included more than one elastographic technique for liver fibrosis assessment in their systems. S8 ultrasound system from General Electric includes TE and 2D-SWE. Philips just launched a system that includes pSWE and 2D-SWE modules. Other systems have available either a pSWE technique (such as Siemens, Esaote), or a 2D-SWE module (Aixplorer, General Electric E10, Canon).

Why would one need two different elastographic methods in the same machine? The EFSUMB Elastography Guidelines [4] stipulate that pSWE is a simple method and that there is no need of previous ultrasound experience for practice, while to perform 2D-SWE experience are needed. On the other hand, the feasibility of different methods is not the same, especially in patients with advanced fibrosis. Thus, having available two different elastographic methods in the same ultrasound system can be an advantage. If one method is not feasible, then the other can be used. In addition, concordant results of both methods increase confidence in the assessment.

In this comparative study between two ARFI based methods (pSWE and 2D-SWE) we observed similar feasibility and almost identical cut-off values, making the system easy to use in clinical practice (and this is important because usually different elastography techniques have different cut-off values). On the other hand, the correlation of these two methods with TE (a validated method) was very good, which creates confidence for the use of both methods in practice.

All ARFI based methods have some advantages as compared to TE: can be performed in a vast majority of cases with only one probe (usually a convex one1-6 $\mathrm{MHz}$ ) can be used in patients with ascites and do not need periodic recalibration (such as TE probes do). For 2D-SWE, 3-5 measurements are enough [4], while for pSWE, 10 or maybe 5 are enough [20].

Our study has some limitations: the number of subjects included is not very large; TE and not liver biopsy was used as the reference; a large proportion of subjects had healthy livers (where elastography is easy to perform), the LS measurements were performed either with $\mathrm{M}$ or XL probes and there were not so many cases of intermediate stages of fibrosis. This is why further studies on larger cohorts of patients are required to validate cut off values for clinical practice.

In conclusion, pSWE and 2D-SWE techniques implemented in the same ultrasound system are feasible methods for assessing liver fibrosis, both techniques strongly correlating with TE results. At the same time, the cut-off values for significant fibrosis and liver cirrhosis for both elastographic methods are similar.

\section{Conflict of interest: none}

\section{References}

1. Seeff LB, Everson GT, Morgan TR, et al. Complication rate of percutaneous liver biopsies among persons with advanced chronic liver disease in the HALT-C trial. Clin Gastroenterol Hepatol 2010;8:877-883.

2. Sporea I. Is there a real future for liver elastography? J Gastrointestin Liver Dis 2012;21:129-131.

3. Imbert-Bismut F, Ratziu V, Pieroni L, et al; MULTIVIRC Group. Biochemical markers of liver fibrosis in patients with hepatitis $\mathrm{C}$ virus infection: a prospective study. Lancet 2001;357:1069-1075.

4. Dietrich CF, Bamber J, Berzigotti A, et al. EFSUMB Guidelines and Recommendations on the Clinical Use of Liver Ultrasound Elastography, Update 2017 (Long Version).Ultraschall Med 2017;38:e16-e47.

5. Ferraioli G, Filice C, Castera L, et al. WFUMB guidelines and recommendations for clinical use of ultrasound elastography: Part 3: liver. Ultrasound Med Biol 2015;41:11611179.

6. European Association for Study of Liver; Asociacion Latinoamericana para el Estudio del Higado.. EASL-ALEH 
Clinical Practice Guidelines: Non-invasive tests for evaluation of liver disease severity and prognosis. J Hepatol. 2015;63:237-264.

7. Sandrin L, Fourquet B, Hasquenoph JM, et al. Transient elastography: a new noninvasive method for assessment of hepatic fibrosis. Ultrasound Med Biol 2003;29:1705-1713.

8. Castera L, Foucher J, Bernard PH, et al. Pitfalls of liver stiffness measurement: a 5-year prospective study of 13,369 examinations. Hepatology 2010;51:828-835.

9. Tsochatzis EA, Gurusamy KS, Ntaoula S, Cholongitas E, Davidson BR, Burroughs AK. Elastography for the diagnosis of severity of fibrosis in chronic liver disease: a metaanalysis of diagnostic accuracy. J Hepatol 2011;54:650659.

10. Samsung Medison S-Shearwave White Paper Recommendation. Samsung Medison Clinical Research Group. Issue Date 26 Apr 2017. Available at https://samsunghealthcare. com.

11. S-Shearwave Imaging ${ }^{\text {TM }}$ Non-invasive quantification of tissue stiffness. Samsung Medison CO., LTD 2018. Available at https://samsunghealthcare.com.

12. Hanley JA, McNeil BJ. The meaning and use of the area under a receiver operating characteristic (ROC) curve. Radiology 1982;143:29-36.

13. Lin LI. A concordance correlation coefficient to evaluate reproducibility. Biometrics 1989;45:255-268.

14. Cassinotto C, Lapuyade B, Mouries A, et al. Noninvasive assessment of liver fibrosis with impulse elastography: comparison of Supersonic Shear Imaging with ARFI and Fibroscan ${ }^{\circledR}$. J Hepatol 2014;61:550-557.

15. Cassinotto C, Boursier J, de Lédinghen V, et al. Liver stiffness in nonalcoholic fatty liver disease: A comparison of supersonic shear imaging, FibroScan, and ARFI with liver biopsy. Hepatology 2016;63:1817-1827.

16. Sporea I, Mare R, Lupuşoru R, et al. Comparative study between four ultrasound Shear Waves Elastographic methods for liver fibrosis assessment. Med Ultrason 2018:30:265271.

17. Ahn SJ, Lee JM, Chang W, et al. Prospective Validation of Intra- and Interobserver Reproducibility of a New Point Shear Wave Elastographic Technique for Assessing Liver Stiffness in Patients with Chronic Liver Disease. Korean J Radiol 2017;18:926-935.

18. Joo I, Kim SY, Park HS, Lee ES, Kang HJ, Lee JM. Validation of a New Point Shear-Wave Elastography Method for Noninvasive Assessment of Liver Fibrosis: A Prospective Multicenter Study. Korean J Radiol 2019;20:1527-1535.

19. Kaposi, PN, Unger, Z, Fejér, B, et al. Interobserver agreement and diagnostic accuracy of shearwave elastography for the staging of hepatitis $\mathrm{C}$ virus-associated liver fibrosis. J Clin Ultrasound 2019 Aug 29. doi:10.1002/jcu.22771.

20. Ferraioli G, De Silvestri A, Reiberger T, et al. Adherence to quality criteria improves concordance between transient elastography and ElastPQ for liver stiffness assessment - A multicenter retrospective study. Dig Liver Dis 2018;50:1056-1061. 